\title{
Les extensomètres longue base souples continûment attachés (ELB-CA), capteurs pour l'analyse vibratoire des structures
}

\author{
Gwendal Cumunel $^{1, a}$, Sylvie Delepine-Lesoille ${ }^{1}$, Pierre Argoul ${ }^{2}$ \\ ET Tien Minh NGUYEN ${ }^{2}$ \\ 1 Division Métrologie et Instrumentation, Laboratoire Central des Ponts et Chaussées (LCPC), 58 boulevard Lefèbvre, \\ 75732 Paris, France \\ 2 Université Paris-Est, Institut Navier, LAMI, École Nationale des Ponts et Chaussée, 6 \& 8 avenue Blaise Pascal, \\ Cité Descartes, Champs-sur-Marne, 77455 Marne la Vallée, France
}

Reçu le 27 octobre 2006, accepté le 29 mai 2007

\begin{abstract}
Résumé - Des Extensomètres souples et de Longue Base (ELB) de mesure sont maintenant disponibles commercialement. Ils sont réalisés avec des fibres optiques interrogées par interférométrie faible cohérence, conditionnées de manière à adhérer continûment à l'ouvrage. Ils sont intéressants pour la surveillance dynamique d'ouvrages d'art car leur longueur significative, vis-à-vis de l'ouvrage étudié, permet d'observer son comportement structurel. L'article s'attache à formuler, de manière analytique, la mesure réalisée par les ELB-CA afin d'anticiper leur réponse. On s'intéresse premièrement au cas du comportement vibratoire linéaire d'une poutre et deuxièmement au cas plus général d'une poutre en grands déplacements. Dans ce deuxième cas, la mesure des ELB, prenant en compte les non-linéarités géométriques, est calculée numériquement. Le calcul analytique des ELB permet alors de montrer les limites de l'hypothèse de linéarité. Des simulations numériques sont présentées pour ces deux cas lors de l'étude dynamique d'une poutre bi-encastrée de type Euler-Bernoulli. Il ressort que la mesure des ELB dépend des non-linéarités géométriques de la structure. En effet, si celles-ci ne sont plus négligeables, les transformées de Fourier des signaux de mesure des ELB contiennent les fréquences propres de la poutre, mais aussi des fréquences supplémentaires, combinaisons de ces fréquences propres.
\end{abstract}

Mots clés : Capteur / fibre optique / non-linéarités géométriques/ grands déplacements / dynamique / structures

\begin{abstract}
Flexible Continuously Attached long gage Fiber Optic Sensors (CA-FOS) as structures vibration analysis sensors. Flexible Continuously Attached long gage Fiber Optic Sensors (CAFOS) are now commercially available. They are optical fiber-based sensor, used as sensing element of a low-cohesion interferometer, fixed to remain connected to the structure. This development enables dynamic monitoring of lineside structures as well as structural behavior. This article intends to analytically formulate the measurements attained by the CA-FOS in order to anticipate their response. The first case of interest is the linear vibrations of the beam and the second the more general case of the beam under large displacements. In this case, CA-FOS measurement, regarding geometric non-linearities, is numerically obtained. Then, the analytic calculations of the CA-FOS allow for proof of the limitations of the linearity hypothesis. Numeric simulations are presented for these two cases with the dynamic study of a FixedFixed Euler-Bernoulli beam. It results that the measurements of the CA-FOS depend on the geometric non-linearities of the structure. In effect, if they are significant, then the signals of the Fourier transformed curves contain the proper frequencies of the beam as well as those of extraneous frequency combinations.
\end{abstract}

Key words: Sensor / optical fiber / geometrical nonlinearity / large rotations / dynamics / structures

\footnotetext{
${ }^{a}$ Auteur pour correspondance : cumunel@lcpc.fr
} 


\section{Nomenclature}

\begin{tabular}{|c|c|}
\hline$A, B:$ & Emplacement d'un miroir partiel dans l'ELB-CA, sans dimension \\
\hline$E:$ & Module d'Young de la poutre, en GPa \\
\hline$F:$ & Amplitude de la force d'excitation $g(x, t)$, en $\mathrm{N}$ \\
\hline$I:$ & Inertie de la section de la poutre, en $\mathrm{m}^{4}$ \\
\hline$L:$ & Longueur de la poutre, en m \\
\hline$M:$ & Point de l'axe neutre de la poutre \\
\hline$N$ : & Nombre de modes propres compris dans la réponse vibratoire de la poutre, sans dimension \\
\hline$O:$ & Origine du repère de coordonnées cartésiennes \\
\hline$P$ : & Point d'une section droite de la poutre situé à la distance $d$ du point de l'axe neutre $M$ \\
\hline$S:$ & Section de la poutre, en $\mathrm{m}^{2}$ \\
\hline$M^{\prime}:$ & Position du point $M$ après déformation de la poutre \\
\hline$P^{\prime}:$ & Position du point $P$ après déformation de la poutre \\
\hline$D_{A B}(t)$ & Mesure physique temporelle de l'ELB-CA entre les points $A$ et $B$, en m \\
\hline$D_{\text {opt }}(t):$ & Mesure optique temporelle de l'ELB-CA entre les points $A$ et $B$, en $\mathrm{m}$ \\
\hline$F_{k}:$ & Projection de la force $g(x, t)$ sur la $k^{\mathrm{e}}$ déformée modale de la poutre \\
\hline$N_{\mathrm{t}}:$ & Nombre de points de l'intervalle de temps discrétisé $\left[t_{0}, t_{\mathrm{f}}\right]$, sans dimension \\
\hline$\Delta D_{A B}:$ & Différence entre la mesure $D_{A B}(t)$ à l'instant $t$ et la mesure à l'instant initial $t=0$, en $\mathrm{s}$ \\
\hline$\Delta D_{\text {opt }}:$ & Différence entre la mesure $D_{\mathrm{opt}}(t)$ à l'instant $t$ et la mesure à l'instant initial $t=0$, en $\mathrm{s}$ \\
\hline$a, b:$ & $\begin{array}{l}\text { Borne d'intégration inférieure (resp. supérieure) de la mesure de l'ELB-CA, à l'emplacement d'un } \\
\text { miroir partiel, en m }\end{array}$ \\
\hline$d:$ & Distance de l'ELB-CA, par rapport à l'axe neutre de la poutre, supposée constante, en m \\
\hline$e:$ & Épaisseur de la poutre, en m \\
\hline$\vec{f}(x, t):$ & Fonction de la courbe paramétrée égale au vecteur déplacement $\overrightarrow{\boldsymbol{O P}^{\prime}}(x, t)$ \\
\hline$g(x, t):$ & Force d'excitation, en $\mathrm{N}$ \\
\hline$h:$ & Hauteur de la poutre, en m \\
\hline$\vec{n}:$ & Vecteur unitaire normal à l'axe neutre de la poutre \\
\hline$n:$ & Composante constante de l'indice de réfraction optique $n_{\mathrm{opt}}$, sans dimension \\
\hline$s:$ & Abscisse curviligne, en m \\
\hline$t:$ & Variable temporelle, en s \\
\hline$u, v:$ & Déplacement axial (resp. transverse) de la poutre, en $\mathrm{m}$ \\
\hline$x, y:$ & Variables d'espace, en m \\
\hline $\overrightarrow{\boldsymbol{f}^{\prime}}(x, t):$ & Dérivée par rapport à $x$ du vecteur déplacement $\overrightarrow{\boldsymbol{O P}}(x, t)$ \\
\hline$u^{\prime}, v^{\prime}:$ & Dérivée par rapport à $x$ du déplacement axial (resp. transverse) de la poutre, sans dimension \\
\hline$u^{\prime \prime}, v^{\prime \prime}:$ & Dérivée seconde par rapport à $x$ du déplacement axial (resp. transverse) de la poutre, en $\mathrm{m}^{-1}$ \\
\hline$b_{k}(t):$ & Réponse de la poutre à la force $F_{k}(t)$, en s \\
\hline$f_{k}:$ & Fréquence du $k^{\mathrm{e}}$ mode de la poutre, en $\mathrm{Hz}$ \\
\hline$n_{\mathrm{opt}}:$ & Indice de réfraction de l'onde optique dans la fibre optique, sans dimension \\
\hline$n_{x}, n_{y}:$ & Composante axiale (resp. transverse) du vecteur $\vec{n}$ normal à l'axe neutre de la poutre \\
\hline$n_{\epsilon}:$ & Composante de $n_{\mathrm{opt}}$ dépendant de la déformation \\
\hline$p_{11}, p_{12}:$ & Constantes photoélastiques, sans dimension \\
\hline$t_{0}:$ & Instant de l'excitation (choc), en s \\
\hline$t_{\mathrm{f}}:$ & Durée du signal de mesure $D_{A B}(t)$, en s \\
\hline$v_{\max }:$ & Flèche maximale de la poutre, en $\mathrm{m}$ \\
\hline$x_{0}:$ & Lieu de l'excitation (choc), en m \\
\hline$\delta:$ & Impulsion de Dirac, sans dimension \\
\hline$\epsilon_{x x}(x, t):$ & Déformation de la poutre suivant l'axe $O x$, en $\mu \epsilon\left(\mu \mathrm{m} . \mathrm{m}^{-1}\right)$ \\
\hline$\xi:$ & Taux d'amortissement choisi constant pour les modes de la poutre, sans dimension \\
\hline$\nu:$ & Coefficient de Poisson de la fibre optique, sans dimension \\
\hline$\rho:$ & Masse volumique de la poutre, en kg.m ${ }^{-3}$ \\
\hline$\alpha_{k}:$ & Constante de normalisation de la $k^{\mathrm{e}}$ déformée modale de la poutre, sans dimension \\
\hline$\beta_{k} L:$ & $k^{\mathrm{e}}$ solution de l'équation transcendante aux valeurs propres de la poutre, sans dimension \\
\hline$\delta_{i j}:$ & Symbole de Kronecker égal à 1 pour $i=j$ et à 0 sinon, sans dimension \\
\hline$\phi_{k}(x)$ & $k^{\mathrm{e}}$ déformée modale de la poutre \\
\hline$\omega_{k}:$ & $k^{\mathrm{e}}$ pulsation propre de la poutre, en rad.s ${ }^{-1}$ \\
\hline$\tilde{\omega}_{k}:$ & $k^{\mathrm{e}}$ pulsation réduite de la poutre, en rad.s ${ }^{-1}$ \\
\hline$\xi_{k}:$ & Taux d'amortissement du $k^{\mathrm{e}}$ mode de la poutre, sans dimension \\
\hline
\end{tabular}




\section{Introduction}

La surveillance d'ouvrages d'art est un domaine actif de la recherche, cependant la mise en place sur ouvrages de l'instrumentation peut s'avérer fastidieuse et coûteuse. En effet, équiper une structure de plusieurs dizaines de mètres nécessite des dizaines de capteurs $[1,2]$, auxquels il faut rajouter le coût de maintenance. C'est pourquoi une autre solution utilisant moins de capteurs est souvent préférée : quelques capteurs (2 ou 3) servant de référence sont fixes et les autres (une dizaine en général) sont déplacés sur l'ensemble de la structure étudiée [3]. Le nombre de capteurs étant réduit, le choix de leur placement peut être assez sensible $[4,5]$. Une alternative possible à ce problème est l'utilisation d'Extensomètres de Longues Bases de mesure (ELB) [6,7]. Réalisés à partir de fibres optiques, des capteurs [8-10] permettent de mesurer des variations de distances entre des points espacés de plusieurs mètres tout en conservant une résolution de l'ordre $\mathrm{du} \mu \mathrm{m}$. Leur utilisation est principalement consacrée à la surveillance des ouvrages à long terme [11,12] mais aussi, plus récemment, à des études dynamiques [12,13]. Une évolution récente permet de les attacher continûment aux structures soit par collage, soit en les noyant au coeur du matériau; nous appellerons ce type de capteur les Extensomètres Longue Base Continûment Attachés (ELBCA) $[14,15]$ aux structures. Ils ont été utilisés pour de la détection de défaut en dynamique [16]. Il a également été démontré que ces capteurs pouvaient servir de filtres modaux [17].

Dans cet article, l'expression mathématique de la mesure réalisée par ce type d'ELB est donnée dans le cas des vibrations d'une poutre droite d'Euler Bernoulli dans le plan $(0, x, y)$ pouvant prendre en compte des nonlinéarités géométriques. Des simulations numériques sont présentées lors de l'étude dynamique d'une poutre biencastrée mise en vibration à l'aide d'un choc.

\section{Description du capteur}

Plusieurs types d'extensomètres à fibres optiques ayant différents principes de mesure existent [18]. Dans notre étude, nous nous référons aux ELB dont le principe de mesure est fondé sur l'interférométrie basse cohérence, pour lesquels le bras de mesure est attaché continûment à la structure et le bras de référence est isolé des déformations subies par la structure [19].

Le système mesure alors le chemin optique entre les miroirs partiels inscrits dans la fibre optique de mesure (d'une certaine longueur arbitraire) désignée sous le nom « capteur ». Comme la fibre optique est un guide d'onde, la mesure correspond à l'intégrale de l'abscisse curviligne et non à la distance euclidienne entre les deux miroirs. Pour déterminer cette distance, l'unité de mesure comprend traditionnellement un photodétecteur, un bras de référence de la même longueur que le capteur, un bras mesurant constitué d'un miroir mobile scannant une certaine distance $X$ de quelques mm, qui correspond en fait à la plage de mesure, et une diode électroluminescente
(LED) dont la lumière est séparée en deux. Une moitié illumine le capteur (bras de mesure), qui réfléchit une partie de la lumière incidente et l'autre moitié illumine une ligne à retard composée du bras de référence et du bras mesurant. Les signaux réfléchis provenant du bras de mesure et de la ligne à retard sont recombinés sur un photodétecteur. Le déplacement du miroir, monté sur un étage motorisé et dont la position est déterminée grâce à une règle optique, permet d'égaliser les chemins optiques du bras de mesure et de la ligne à retard et d'obtenir l'information de distance. Quand le temps de décalage entre les deux réflexions aux extrémités de la cavité (miroirs partiels) est reproduit dans la ligne à retard, un pic d'interférence apparaît [19,20] (Fig. 1). La détermination est absolue (et non à $2 \pi$ près) car la source lumineuse est choisie peu cohérente : au-delà de la longueur de cohérence de la source, c'est-à-dire quelques $\mu \mathrm{m}$, les franges d'interférences sont brouillées [20]. Connaissant précisement la position du miroir, la longueur de la cavité peut alors être déterminée. Tout changement de longueur du capteur correspondra à une nouvelle position du miroir pour laquelle les interférences sont constructives (c'est-à-dire à une nouvelle distance de la ligne à retard).

Lorsque la structure subit des déformations, que ce soit en traction ou en compression, la fibre collée ou incorporée à la structure s'allonge ou se rétracte en conséquence. La mesure ainsi obtenue est la distance entre les miroirs le long de la fibre optique. On suppose que les effets thermiques sont corrigés à l'aide d'une jauge témoin ou avec d'autres moyens de compensation thermique (par exemple en plaçant le bras de référence dans la même ambiance thermique que le bras de mesure). Dans l'exemple montré sur la figure 1, cinq pics d'interférence sont détectés pour cinq cavités montées en série.

\section{Calcul théorique de la mesure réalisée par I'ELB-CA}

Comme décrit précédemment, l'ELB-CA permet de mesurer la distance entre deux points particuliers le long de la fibre. Plus précisément, la mesure temporelle théorique réalisée par l'ELB-CA est (principe de Fermat [20]) :

$$
D_{\mathrm{opt}}(t)=\int_{s_{A}}^{s_{B}} n_{\mathrm{opt}} \mathrm{d} s
$$

où $n_{\mathrm{opt}}$ est l'indice de réfraction (rapport de la vitesse de propagation de la lumière dans le matériau par rapport au vide), $D_{\text {opt }}(t)$ la longueur optique mesurée par le capteur, $A$ et $B$ sont les points où les miroirs du capteur sont situés, d'abscisses respectives $s_{A}$ et $s_{B} ; s$ représentant l'abscisse curviligne.

Dans une première approximation, l'indice de réfraction sera considéré comme étant constant (pour une fibre optique monomode, $\left.n_{\mathrm{opt}} \approx 1,46\right)$. Alors l'équation (1) devient

$$
D_{\mathrm{opt}}(t)=n_{\mathrm{opt}} \cdot D_{A B}(t)
$$




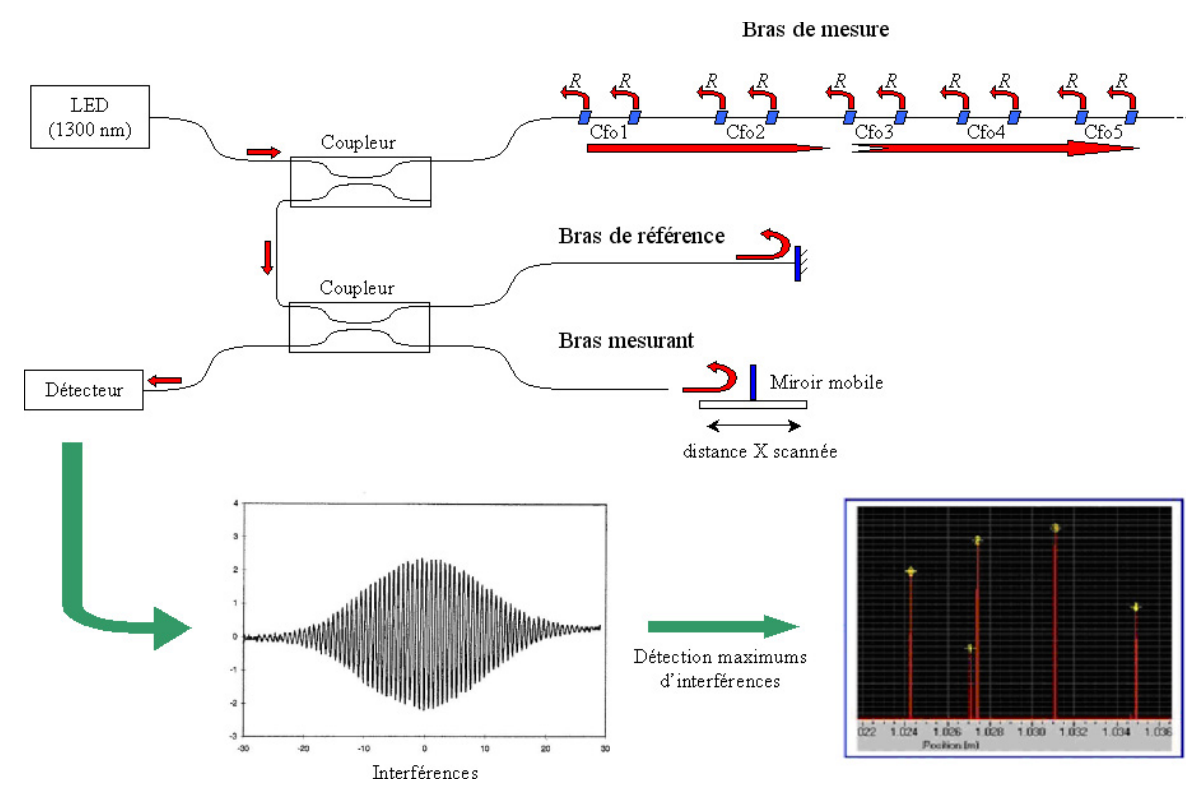

Fig. 1. Principe de l'interféromètre basse cohérence pour mesurer la longueur des cavités optiques du bras de mesure.

avec

$$
D_{A B}(t)=\int_{s_{A}}^{s_{B}} \mathrm{~d} s
$$

où $D_{A B}(t)$ est la longueur physique du capteur.

L'influence de la dépendance de l'indice de réfraction avec la déformation, mise en évidence dans [19,21,22], est abordée en section 2.2.

La longueur optique $D_{\text {opt }}(t)$ étant égale à la longueur physique $D_{A B}(t)$ à une constante multiplicative près, nous allons nous intéresser uniquement au calcul de $D_{A B}(t)$. L'exemple d'application choisi pour l'étude concerne les vibrations d'une poutre droite dans le plan $(O, x, y)$ avec les hypothèses cinématiques d'EulerBernoulli : une section droite de la poutre reste plane et perpendiculaire à l'axe neutre au cours de la déformation.

Le champ de déplacement de l'axe neutre est défini dans le repère galiléen $(O, \vec{i}, \vec{j}, \overrightarrow{\boldsymbol{k}})$ par :

$$
\overrightarrow{\boldsymbol{O M}}(x, 0,0) \rightarrow \overrightarrow{\boldsymbol{O M}}^{\prime}(x+u(x, t), v(x, t), 0)
$$

où $u$ et $v$ sont respectivement les déplacements axial et transverse de la poutre.

Un point $P$ situé à une distance $d$, supposée constante, de l'axe neutre se déplacera en $P^{\prime}$ (Fig. 2) :

$$
\overrightarrow{\boldsymbol{O P}}(x, d, 0) \rightarrow \overrightarrow{\boldsymbol{O P}}^{\prime}\left(X^{\prime}, Y^{\prime}, 0\right)
$$

avec

$$
\begin{aligned}
\overrightarrow{\boldsymbol{O P}^{\prime}}(x, t) & =\overrightarrow{\boldsymbol{O M}}(x, t)+\overrightarrow{\boldsymbol{M}^{\prime}}(x, t)+\overrightarrow{\boldsymbol{M}^{\prime} \boldsymbol{P}^{\prime}}(x, t) \\
& =\left|\begin{array}{l}
X^{\prime} \\
Y^{\prime} \\
0
\end{array}=\right| \begin{array}{l}
x+u(x, t)+d \cdot n_{x}(x, t) \\
v(x, t)+d \cdot n_{y}(x, t) \\
0
\end{array}
\end{aligned}
$$

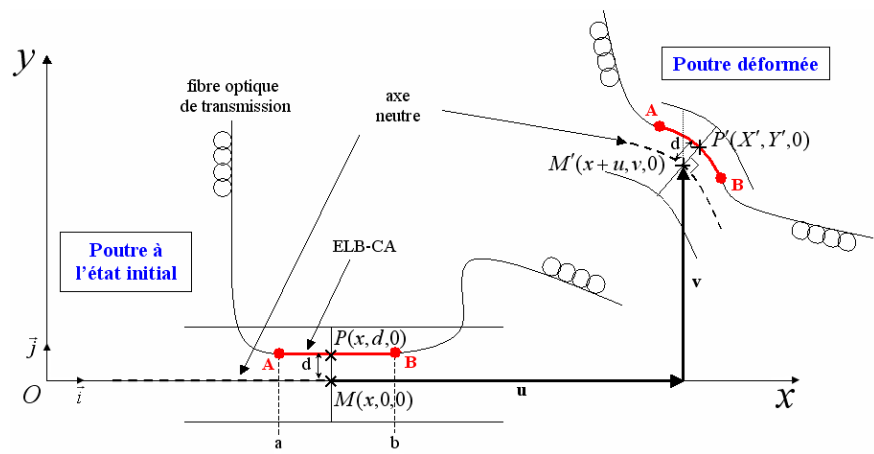

Fig. 2. Déformation d'une section droite transverse de la poutre.

où $n_{x}$ et $n_{y}$ sont respectivement les composantes axiale et transverse du vecteur normal à l'axe neutre $\overrightarrow{\boldsymbol{n}}$ défini par :

$$
\overrightarrow{\boldsymbol{n}}=\left|\begin{array}{l}
n_{x} \\
n_{y} \\
0
\end{array}=\frac{1}{|\overrightarrow{\boldsymbol{k}} \wedge \overrightarrow{\boldsymbol{t}}|} \overrightarrow{\boldsymbol{k}} \wedge \overrightarrow{\boldsymbol{t}}=\frac{1}{\sqrt{\left(1+u^{\prime}\right)^{2}+v^{\prime 2}}}\right| \begin{aligned}
& -v^{\prime} \\
& 1+u^{\prime} \\
& 0
\end{aligned}
$$

avec $\overrightarrow{\boldsymbol{t}}=\frac{1}{\sqrt{\left(1+u^{\prime}\right)^{2}+v^{\prime 2}}} \mid \begin{aligned} & 1+u^{\prime} \\ & v^{\prime} \\ & 0\end{aligned}$, le vecteur tangent à l'axe neutre.

Pour des soucis de clarté dans les expressions, nous avons omis les variables $x$ et $t$. Elles ne seront plus mentionnées et nous aurons alors $u(x, t)=u, v(x, t)=v$, $\frac{\mathrm{d} u}{\mathrm{~d} x}(x, t)=u^{\prime}$ et $\frac{\mathrm{d} v}{\mathrm{~d} x}(x, t)=v^{\prime}$, il en sera de même pour les dérivées secondes.

Pour déterminer la mesure $D_{A B}(t)$, on considère que tous les points de la poutre sont le support d'un arc paramétré, i.e. ils décrivent une courbe paramétrée représentée par la fonction $\vec{f}=\overrightarrow{\boldsymbol{O P}^{\prime}}$, si bien que 


$$
D_{A B}(t)=\int_{a}^{b} \sqrt{\left(1+u^{\prime}\right)^{2}+v^{\prime 2}+2 d \frac{u^{\prime \prime} v^{\prime}-\left(1+u^{\prime}\right) v^{\prime \prime}}{\sqrt{\left(1+u^{\prime}\right)^{2}+v^{\prime 2}}}+d^{2} \frac{\left[u^{\prime \prime} v^{\prime}-\left(1+u^{\prime}\right) v^{\prime \prime}\right]^{2}}{\left[\left(1+u^{\prime}\right)^{2}+v^{\prime 2}\right]^{2}}} \mathrm{~d} x
$$

$$
\begin{cases}\rho S \ddot{v}+E I v^{\prime \prime \prime \prime}-E S\left[\left(u^{\prime}+\frac{1}{2} v^{2}\right) v^{\prime \prime}+\left(u^{\prime \prime}+v^{\prime} v^{\prime \prime}\right) v^{\prime}\right] & =F_{v} \\ \rho S \ddot{u}-E S\left(u^{\prime \prime}+v^{\prime} v^{\prime \prime}\right) & =F_{u}\end{cases}
$$

la mesure réalisée par l'ELB-CA est tout simplement la longueur de cet arc entre les deux points $A$ et $B$ :

$$
D_{A B}(t)=\int_{a}^{b}\left\|\overrightarrow{\boldsymbol{f}}^{\prime}(x, t)\right\| \mathrm{d} x
$$

Finalement, après calcul, la mesure réalisée par l'ELBCA s'écrit :

$$
\text { voir l'équation (9) ci-dessus }
$$

On introduit souvent la courbure $C$ définie par $C=$ $\frac{1}{r}=\left|\frac{\mathrm{d} \alpha}{\mathrm{d} s}\right|$ où $r$ est le rayon de courbure et $\alpha$ l'angle que fait l'axe $O x$ avec la tangente en $M$. Son expression est ici $[25]$ :

$$
C=\frac{\left[v^{\prime \prime}\left(1+u^{\prime}\right)-u^{\prime \prime} v^{\prime}\right]}{\left[\left(1+u^{\prime}\right)^{2}+v^{\prime 2}\right]^{\frac{3}{2}}}
$$

\subsection{Hypothèses simplificatrices}

Plusieurs hypothèses seront successivement et/ou conjointement effectuées afin de simplifier l'équation (9), c'est-à-dire déterminer les termes prépondérants de l'expression :

H1 : La toute première hypothèse réalisée est celle des petites déformations. Vues les hypothèses de notre étude, vibrations de la poutre dans le plan $(O, x, y)$, la déformation au niveau de l'ELB-CA se limite au terme $\epsilon_{x x}$ (déformation d'un fil) et la variation d'abscisse curviligne d $s$ est alors [24] :

$$
\mathrm{d} s^{2}=\mathrm{d} s_{0}^{2}+2 \epsilon_{x x} \mathrm{~d} x^{2}
$$

avec $\mathrm{d} s_{0}$, la variation d'abscisse curviligne à l'instant initial, qui sera égale à dx (1'ELB-CA étant droit à l'origine), d'où :

$$
\mathrm{d} s=\sqrt{1+2 \epsilon_{x x}} \mathrm{~d} x
$$

En rapprochant la relation précédente (12) de celle obtenue pour la mesure de la longueur de l'arc $\widehat{A B}(9)$, on déduit l'expression de la déformation $\epsilon_{x x}:$

$$
\begin{aligned}
\epsilon_{x x}=u^{\prime}+\frac{u^{\prime 2}}{2}+\frac{v^{\prime 2}}{2} & +d \frac{u^{\prime \prime} v^{\prime}-\left(1+u^{\prime}\right) v^{\prime \prime}}{\sqrt{\left(1+u^{\prime}\right)^{2}+v^{\prime 2}}} \\
+ & \frac{d^{2}}{2} \frac{\left[u^{\prime \prime} v^{\prime}-\left(1+u^{\prime}\right) v^{\prime \prime}\right]^{2}}{\left[\left(1+u^{\prime}\right)^{2}+v^{\prime 2}\right]^{2}}
\end{aligned}
$$

On vérifie aisément que l'on retrouve la même expression de la déformation à partir du vecteur déplacement $\overrightarrow{\boldsymbol{P P}^{\prime}}=\overrightarrow{\boldsymbol{O P}^{\prime}}-\overrightarrow{\boldsymbol{O P}}=\mid \begin{aligned} & X^{\prime}-x \\ & Y^{\prime}-d \text { dont } \\ & 0\end{aligned}$ les composantes $X^{\prime}$ et $Y^{\prime}$ sont données en (6).

Par suite, pour l'exemple d'application choisi (poutre droite), la mesure $D_{A B}(t)$ du capteur peut s'exprimer en fonction de la déformation :

$$
D_{A B}(t)=\int_{a}^{b} \sqrt{1+2 \epsilon_{x x}} \mathrm{~d} x
$$

Comme on fait l'hypothèse des petites déformations $\left(\epsilon_{x x} \ll 1\right)$, il est possible d'effectuer un développement limité au premier ordre de l'expression (14) :

$$
D_{A B}(t) \approx \int_{a}^{b}\left(1+\epsilon_{x x}\right) \mathrm{d} x
$$

soit

$$
\Delta D_{A B}(t)=D_{A B}(t)-D_{A B}(0)=\int_{a}^{b} \epsilon_{x x} \mathrm{~d} x
$$

avec $D_{A B}(0)=\int_{a}^{b} \mathrm{~d} x=b-a$.

Cette hypothèse $\mathrm{H} 1$, des petites déformations, sera appliquée dans la suite de l'article.

H2 : Équations du mouvement de la poutre couplées et non-linéaires : Cas Général (Cas G).

Dans ce cas le plus général, la mesure de l'ELB-CA sera déterminée à partir des déplacements axial et transverse de la poutre, issus de la résolution des équations d'équilibre « classiques » régissant la vibration des poutres en grands déplacements :

$$
\text { voir l'équation (17) ci-dessus }
$$

Les termes $u^{\prime}, u^{\prime \prime}, v^{\prime}$ et $v^{\prime \prime}$ seront déterminés à partir de ce système d'équations et réinjectés dans l'expression de la mesure de l'ELB-CA. Deux cas sont étudiés :

H2.1 : ELB-CA situé à une hauteur $d$ par rapport à l'axe neutre.

La variation de la mesure physique de l'ELBCA $\Delta D_{A B}(t)$ s'écrit :

$$
\begin{aligned}
\Delta D_{A B}(t)= & \frac{1}{2} \int_{a}^{b}\left(2 u^{\prime}+u^{\prime 2}+v^{\prime 2}\right. \\
& +2 d \frac{u^{\prime \prime} v^{\prime}-\left(1+u^{\prime}\right) v^{\prime \prime}}{\sqrt{\left(1+u^{\prime}\right)^{2}+v^{\prime 2}}} \\
& \left.+d^{2} \frac{\left[u^{\prime \prime} v^{\prime}-\left(1+u^{\prime}\right) v^{\prime \prime}\right]^{2}}{\left[\left(1+u^{\prime}\right)^{2}+v^{\prime 2}\right]^{2}}\right) \mathrm{d} x
\end{aligned}
$$


H2.2 : ELB-CA situé au niveau de l'axe neutre $(d=$ $0)$

Le cas de l'axe neutre de la poutre correspond au cas particulier de la poutre pour lequel la distance $d$ est nulle. Alors, à partir des équations (13) et (16), la mesure $\Delta D_{A B}(t)$ du capteur devient :

$$
\Delta D_{A B}(t)=\frac{1}{2} \int_{a}^{b}\left(2 u^{\prime}+u^{\prime 2}+v^{\prime 2}\right) \mathrm{d} x
$$

H3 : Équations du mouvement de la poutre découplées : Cas Linéaire (Cas L)

Le système d'équations est alors :

$$
\left\{\begin{array}{l}
\rho S \ddot{v}+E I v^{\prime \prime \prime \prime}=F_{v} \\
\rho S \ddot{u}-E S u^{\prime \prime}=F_{u}
\end{array}\right.
$$

L'étude présentée porte sur les vibrations de flexion de la poutre, c'est-à-dire l'équation linéaire :

$$
\rho S \ddot{v}+E I v^{\prime \prime \prime \prime}=F_{v}
$$

Notons par ailleurs que l'excitation est uniquement transverse $\left(F_{u}=0\right)$ et que, par conséquent, le déplacement axial est nul. Dans l'expression de la mesure $D_{A B}(t)$, seuls les termes en $v$ seront conservés. Les deux mêmes cas que pour l'hypothèse H2 sont étudiés :

H3.1 : ELB-CA situé à une hauteur $d$ par rapport à l'axe neutre

La mesure de l'ELB-CA devient alors

$$
\begin{array}{r}
\Delta D_{A B}(t)=\frac{1}{2} \int_{a}^{b}\left(v^{\prime 2}-2 d \frac{v^{\prime \prime}}{\sqrt{1+v^{\prime 2}}}\right. \\
\left.+d^{2} \frac{v^{\prime \prime 2}}{\left[1+v^{\prime 2}\right]^{2}}\right) \mathrm{d} x
\end{array}
$$

et l'équation (10) :

$$
C=\frac{v^{\prime \prime}}{\left[1+v^{\prime 2}\right]^{\frac{3}{2}}}
$$

H3.2 : ELB-CA situé au niveau de l'axe neutre $(d=$ 0)

Pour la mesure de l'ELB-CA situé au niveau de l'axe neutre, $d=0$, il vient :

$$
\Delta D_{A B}(t)=\frac{1}{2} \int_{a}^{b} v^{\prime 2} \mathrm{~d} x
$$

On remarque à partir des hypothèses $\mathrm{H} 2$ et $\mathrm{H} 3$ que la mesure de l'ELB-CA situé initialement à une distance $d$ de l'axe neutre peut s'exprimer en fonction de la mesure de l'ELB-CA situé au niveau de l'axe neutre et d'un terme dépendant de $d$, distance à l'axe neutre :

$$
\Delta D_{A B}(t)=\Delta D_{A B}^{\mathrm{axe}}+D_{A B}^{d}(t)
$$

où

$$
\begin{aligned}
D_{A B}^{d}(t)= & \frac{1}{2} \int_{a}^{b}\left(2 d \frac{u^{\prime \prime} v^{\prime}-\left(1+u^{\prime}\right) v^{\prime \prime}}{\sqrt{\left(1+u^{\prime}\right)^{2}+v^{\prime 2}}}\right. \\
& \left.+d^{2} \frac{\left[u^{\prime \prime} v^{\prime}-\left(1+u^{\prime}\right) v^{\prime \prime}\right]^{2}}{\left[\left(1+u^{\prime}\right)^{2}+v^{\prime 2}\right]^{2}}\right) \mathrm{d} x \quad \text { pour le cas G. } \\
D_{A B}^{d}(t)= & -\frac{1}{2} \int_{a}^{b}\left(2 d \frac{v^{\prime \prime}}{\sqrt{1+v^{\prime 2}}}-d^{2} \frac{v^{\prime \prime 2}}{\left[1+v^{\prime 2}\right]^{2}}\right) \mathrm{d} x \\
& \text { pour le cas L. }
\end{aligned}
$$

En appliquant la transformée de Fourier (opérateur linéaire) à (25), il vient :

$$
T F\left(\Delta D_{A B}\right)(\omega)=T F\left(\Delta D_{A B}^{\mathrm{axe}}\right)(\omega)+T F\left(D_{A B}^{d}\right)(\omega)
$$

\subsection{Raffinement du modèle optique de la mesure}

La dépendance de l'indice de réfraction optique $n_{\mathrm{opt}}$ avec la déformation a été jusqu'ici négligée. Lorsqu'elle est prise en compte, l'expression de la mesure réalisée par l'ELB-CA est :

$$
D_{\mathrm{opt}}(t)=\int_{a}^{b} n_{\mathrm{opt}} \mathrm{d} s=\int_{a}^{b}\left(n+n_{\epsilon}\right) \mathrm{d} s
$$

où $n=1,46$ et $n_{\epsilon}=-\frac{1}{2} n^{3}\left[(1-\nu) p_{12}-\nu p_{11}\right] \epsilon_{x x}=\kappa \epsilon_{x x}$, défini en [19], est la partie de l'indice dépendant de la déformation. Les constantes mécaniques de la fibre sont données dans [19] : le coefficient de Poisson $\nu=0,25$ et les constantes photoélastiques $p_{11} \approx 0,12$ et $p_{12} \approx 0,27$, ce qui donne $\kappa=-0,26$.

À partir de l'équation (15), l'expression de la mesure s'écrit alors :

$$
\begin{aligned}
D_{\text {opt }}(t) & =\int_{a}^{b} n_{\text {opt }}\left(1+\epsilon_{x x}\right) \mathrm{d} x \\
& =\int_{a}^{b}\left(n+\kappa \epsilon_{x x}\right)\left(1+\epsilon_{x x}\right) \mathrm{d} x \\
& =n \int_{a}^{b} \mathrm{~d} x+n \int_{a}^{b}\left[1+\frac{\kappa}{n}\right] \epsilon_{x x} \mathrm{~d} x+\kappa \int_{a}^{b} \epsilon_{x x}^{2} \mathrm{~d} x
\end{aligned}
$$

et en négligeant les termes en $\epsilon_{x x}^{2}$ (hypothèses des petites déformations), on obtient :

$$
\Delta D_{\mathrm{opt}}(t)=n \int_{a}^{b}\left[1+\frac{\kappa}{n}\right] \epsilon_{x x} \mathrm{~d} x
$$

avec $\Delta D_{\text {opt }}(t)=D_{\text {opt }}(t)-D_{\text {opt }}(0)$ et $D_{\text {opt }}(0)=n \int_{a}^{b} \mathrm{~d} x=$ $n(b-a)$.

Lorsque $n_{\mathrm{opt}}=n$, c'est-à-dire $n_{\epsilon}=0$, la mesure optique réalisée par l'ELB-CA (cf. Éqs. (2) et (15)) est :

$$
D_{\text {opt }}(t)=n D_{A B}(t)=n \int_{a}^{b}\left(1+\epsilon_{x x}\right) \mathrm{d} x
$$

d'où

$$
\Delta D_{\text {opt }}(t)=n \int_{a}^{b} \epsilon_{x x} \mathrm{~d} x
$$

En comparant les expressions (29) et (31) obtenues pour la mesure optique $\Delta D_{\text {opt }}$, on s'aperçoit que la 
dépendance de l'indice de réfraction avec la déformation a une influence sur l'amplitude du signal temporel et de la TF, mais pas sur son contenu fréquentiel, qui est étudié par la suite dans la partie 3 .

Enfin, pour être exhaustif, il faut prendre en considération l'influence d'une éventuelle variation de température lors de la sollicitation dynamique de la poutre, à la fois sur l'allongement propre du capteur et sur la dépendance de l'indice de réfraction avec la température [26]. Toutefois la dynamique des phénomènes thermiques étant beaucoup plus lente que celle des phénomènes vibratoires étudiés, son influence reste négligeable pour notre étude. D'autre part, la prise en compte de l'effet thermique est très dépendante de la façon dont la correction thermique est effectuée, par exemple avec l'association d'un extensomètre témoin [26,27] ou un capteur de type différent tel un capteur à réseau de Bragg [28]. En conséquence, elle ne peut être effectuée que pour un système expérimental précis, ce qui est hors de propos ici.

\subsection{Lien avec l'état de l'art}

Habituellement dans la littérature [11,25], la mesure de l'ELB est utilisée pour déterminer la courbure moyenne $C_{\text {moy }}$, qui est exprimée dans [11], pour le cas de la flexion simple d'une poutre d'Euler-Bernoulli, par :

$$
\begin{aligned}
C_{\mathrm{moy}}= & \frac{1}{L_{\mathrm{ELB}}} \int_{0}^{L_{\mathrm{ELB}}} C(x) \mathrm{d} x= \\
& \frac{1}{d \cdot L_{\mathrm{ELB}}} \int_{0}^{L_{\mathrm{ELB}}} \varepsilon_{x x}(x) \mathrm{d} x=-\frac{\Delta L_{\mathrm{ELB}}}{d \cdot L_{\mathrm{ELB}}}
\end{aligned}
$$

avec

$$
\Delta L_{\mathrm{ELB}}(t)=\int_{0}^{L_{\mathrm{ELB}}} \varepsilon_{x x} \mathrm{~d} x=-d \int_{0}^{L_{\mathrm{ELB}}} v^{\prime \prime} \mathrm{d} x \text { et } C=v^{\prime \prime}
$$

où $\varepsilon_{x x}$ est la déformation axiale de la poutre, $\Delta L_{\mathrm{ELB}}$ l'élongation de l'ELB $\left(=L_{\mathrm{ELB}}(t)-L_{\mathrm{ELB}}\right)$ et $L_{\mathrm{ELB}}$ la longueur de l'ELB à l'origine.

Nous allons montrer à partir des résultats obtenus précédemment en sections 2.1 et 2.2 que cette formule n'est valable que sous certaines hypothèses.

Pour retrouver les expressions de la déformation et de la courbure définies en (33) à partir de celles définies en (13) et (10), il faut dans un premier temps se placer dans le cas L (équations découplées) :

$$
\epsilon_{x x}=\frac{v^{\prime 2}}{2}-d \frac{v^{\prime \prime}}{\sqrt{1+v^{\prime 2}}}+\frac{d^{2}}{2} \frac{v^{\prime \prime 2}}{\left[1+v^{\prime 2}\right]^{2}} \text { et } C=\frac{v^{\prime \prime}}{\left[1+v^{\prime 2}\right]^{\frac{3}{2}}}
$$

et ensuite faire les hypothèses suivantes : $v^{\prime \prime}<<1, v^{\prime}<<$ 1 et $\frac{v^{\prime 2}}{2}<<d v^{\prime \prime}$. Il vient alors

$$
\epsilon_{x x}=-d v^{\prime \prime} \text { et } C=v^{\prime \prime}
$$

On remarque alors que pour le cas d'un ELB situé au niveau de l'axe neutre $(d=0)$, la déformation est nulle et donc la mesure de l'ELB l'est également.
Par ailleurs, la mesure en sortie du capteur est d'après (29) :

$$
\Delta D_{\mathrm{opt}}(t)=-d(n+\kappa) \int_{a}^{b} C(x) \mathrm{d} x
$$

L'expression (34) peut alors s'exprimer en fonction de la courbure moyenne :

$$
\Delta D_{\mathrm{opt}}(t)=-d(n+\kappa)(b-a) C_{\mathrm{moy}}
$$

ce qui nous permet de faire l'analogie avec l'expression de la courbure moyenne (32) issue de la littérature :

$$
\begin{aligned}
C_{\text {moy }}=\frac{1}{b-a} & \int_{a}^{b} C(x) \mathrm{d} x= \\
& \quad-\frac{\Delta D_{\mathrm{opt}}}{d \cdot D_{\mathrm{opt}}(0)} \cdot \frac{n}{n+\kappa}=-\frac{n}{n+\kappa} \cdot \frac{\Delta L}{d \cdot L}
\end{aligned}
$$

où $\Delta D_{\mathrm{opt}}=D_{\mathrm{opt}}(t)-D_{\mathrm{opt}}(0)$ et $D_{\mathrm{opt}}(0)=n(b-a)$.

Ainsi, les calculs généralement effectués par les utilisateurs des ELB sont valides pour les équations « découplées », avec de «faibles » amplitudes de déformation et sans la prise en compte de la dépendance de l'indice de réfraction optique avec la déformation.

\section{Simulations numériques}

\subsection{Cas linéaire}

L'hypothèse H3 est effectuée pour le calcul numérique de la mesure de l'ELB-CA dans cette partie. Nous allons étudier deux positions particulières du capteur sur la poutre : l'ELB-CA situé à une distance $d=h / 2$ de l'axe neutre (hyp. H3.1), c'est-à-dire collé à la surface de la poutre, et l'ELB-CA situé au niveau de l'axe (hyp. H3.2). Les exemples traités numériquement concernent les réponses vibratoires d'une poutre d'Euler-Bernoulli biencastrée (E-E) à un choc.

La poutre est homogène de section constante rectangle $(S=h \cdot e)$ et de longueur $L$. La réponse pour le déplacement transverse de la poutre à un choc suivant $\vec{j}$, lorsque seuls les $N$ premiers modes sont pris en compte, est donnée classiquement par superposition modale (cas linéaire) [24] :

$$
v(x, t) \approx \sum_{k=1}^{N} b_{k}(t) \phi_{k}(x)
$$

où $b_{k}(t)$ et $\phi_{k}(x)$ sont respectivement la réponse à la force $g(x, t)$ et les déformées modales de la poutre. $g(x, t)$ est la force appliquée à la poutre, à l'abscisse $x_{0}$ et à l'instant $t_{0}$. Elle sera donc définie par $: g(x, t)=F \cdot \delta\left(t-t_{0}\right) \cdot \delta\left(x-x_{0}\right)$.

L'expression de la réponse à la force $g(x, t)$ est :

$$
b_{k}(t)=\frac{1}{\rho S \tilde{\omega_{k}}} \int_{0}^{t} F_{k}(\tau) e^{-\xi_{k} \omega_{k}(t-\tau)} \cdot \sin \left(\tilde{\omega_{k}}(t-\tau)\right) \mathrm{d} \tau
$$


Tableau 1. Paramètres de la poutre.

\begin{tabular}{cccccc}
\hline \multicolumn{2}{l}{ Longueur Hauteur Épaisseur $\begin{array}{l}\text { Module } \\
\text { d'Young }\end{array}$} & Densité & Inertie \\
\hline$L(\mathrm{~m})$ & $h(\mathrm{~m})$ & $e(\mathrm{~m})$ & $E(\mathrm{GPa})$ & $\rho\left(\mathrm{kg} \cdot \mathrm{m}^{-3}\right)$ & $I\left(\mathrm{~m}^{4}\right)$ \\
\hline 1,5 & 0,04 & 0,003 & 210 & 7800 & $9 \times 10^{-11}$ \\
\hline
\end{tabular}

où $F_{k}(t)=\int_{0}^{L} g(x, t) \cdot \phi_{k}(x) \mathrm{d} x, \quad \tilde{\omega}_{k}=\omega_{k} \sqrt{1-\xi_{k}^{2}}$ et $\omega_{k}=\frac{\left(\beta_{k} L\right)^{2}}{L^{2}} \sqrt{\frac{E I}{\rho S}}$.

$\beta_{k} L$ est une solution de l'équation transcendante aux valeurs propres correspondant à la condition aux limites étudiée (poutre E-E) :

$$
\cos \left(\beta_{k} L\right) \cdot \cosh \left(\beta_{k} L\right)=1
$$

L'expression des déformées modales pour la condition aux limites E-E est [24] :

$$
\begin{aligned}
\phi_{k}=\frac{\alpha_{k}}{\sqrt{\rho S L}}[ & \frac{\cos \left(\beta_{k} L\right)-\cosh \left(\beta_{k} L\right)}{\sinh \left(\beta_{k} L\right)-\sin \left(\beta_{k} L\right)}\left(\sinh \left(\beta_{k} x\right)\right. \\
& \left.\left.-\sin \left(\beta_{k} x\right)\right)+\cosh \left(\beta_{k} x\right)-\cos \left(\beta_{k} x\right)\right]
\end{aligned}
$$

où $\alpha_{k}$ est introduit pour normaliser les déformées modales par rapport à la masse :

$$
\int_{0}^{L} \rho S \phi_{i}(x) \phi_{j}(x) \mathrm{d} x=\delta_{i j}
$$

avec $\delta_{i j}$ le symbole de Kronecker : $\delta_{i j}=1$ si $i=j$ et 0 sinon.

Les valeurs des paramètres choisis pour l'étude numérique sont répertoriées dans les tableaux 1 et 2 .

Un des objectifs de notre travail est d'évaluer les limites de validité de l'expression de la mesure issue de l'état de l'art (33). Dans une première étape, nous avons reporté l'expression de la flèche de la poutre $v(x, t)(37)$, obtenue dans le cas linéaire, dans les approximations (22) (ou (24) selon la position de l'ELB) et (33).

On peut noter que pour des niveaux de flèche de la poutre importants, l'équation (37) n'est plus valide et l'équation différentielle régissant le comportement de la poutre en vibration de flexion n'est plus linéaire (17). Néanmoins, nous utilisons cette équation dans cette étape quelle que soit la valeur atteinte pour la flèche maximale de la poutre. La mesure de L'ELB-CA déterminée à partir du système (17) sera étudiée en section 3.2.

\subsubsection{ELB-CA situé à la surface $(d=h / 2)$}

Les mesures simulées de l'ELB-CA sont obtenues numériquement à partir des équations (22) et (33); leur TF est ensuite calculée. Les mesures sont effectuées entre les instants $t_{0}=0$ et $t_{\mathrm{f}}$, intervalle de temps discrétisé en $N_{\mathrm{t}}$ points. Les TF sont tracées sur la plage de fréquence [0-50] Hz. Deux cas sont envisagés suivant deux valeurs (a)

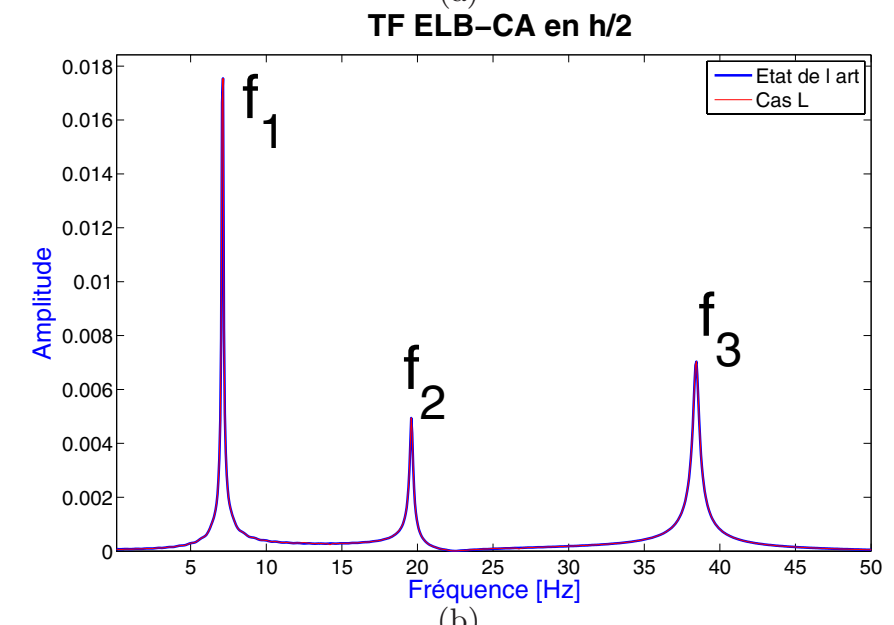

TF ELB-CA en $h / 2$

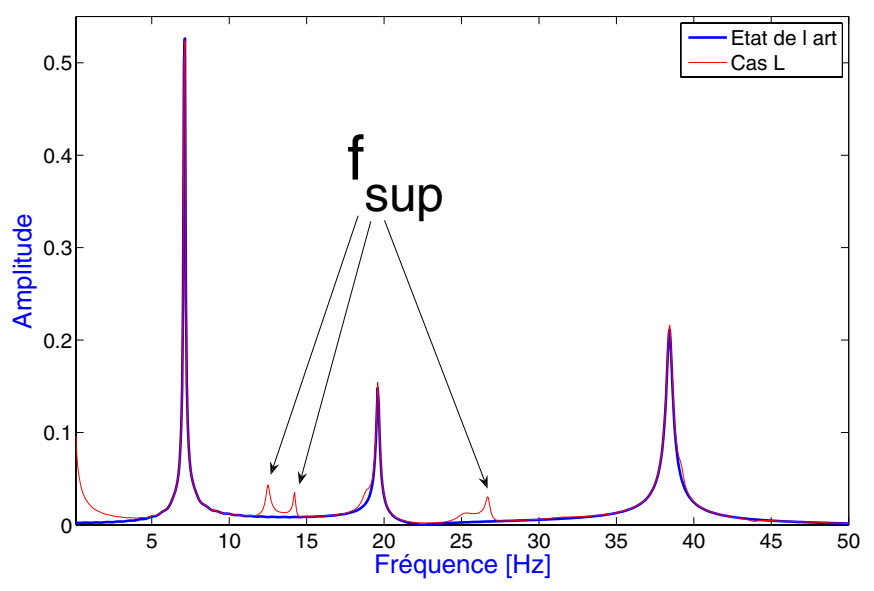

Fig. 3. Comparaison entre la mesure analytique déterminée en (22) pour le cas L et la mesure issue de l'état de l'art (33) : TF d'un ELB-CA situé à la surface de la poutre $(d=h / 2)$ pour une flèche maximale de la poutre (a) $v_{\max }=5 \times 10^{-5} \mathrm{~m}$ et (b) $v_{\max }=1,5 \times 10^{-3} \mathrm{~m}$.

différentes de la flèche maximale $v_{\max }$ de la poutre E-E. La figure 3 a est obtenue pour $v_{\max }=5 \times 10^{-5} \mathrm{~m}$ (faible flèche de la poutre) et la figure $3 \mathrm{~b}$ pour $v_{\max }=1,5 \times 10^{-3} \mathrm{~m}$ (flèche de la poutre plus importante).

Les conclusions tirées des graphes de la figure 3 sont :

i) Dans le cas de «faibles » flèches, les formules approchées utilisées dans la littérature [11, 25] permettent d'obtenir un résultat identique à celui obtenu par la relation (22) correspondant à la mesure $D_{A B}(t)$ pour le cas L (les deux courbes sont confondues, cf. Fig. 3a).

ii) Pour des flèches «plus importantes », c'est-à-dire lorsque les non-linéarités géométriques ne sont plus négligeables, des fréquences supplémentaires apparaissent en complément des fréquences propres de la structure. Ces fréquences additionnelles, répertoriées dans le tableau 3, sont des combinaisons linéaires des fréquences propres de la structure (par exemple : $2 f_{1}$, 
Tableau 2. Paramètres de la simulation.

\begin{tabular}{cccccccc}
\hline $\begin{array}{c}\text { Abscisse } \\
\text { du choc }\end{array}$ & $\begin{array}{c}\text { Instant } \\
\text { du choc }\end{array}$ & $\begin{array}{c}\text { Instant } \\
\text { final des } \\
\text { mesures }\end{array}$ & $\begin{array}{c}\text { Nombre } \\
\text { de pas de } \\
\text { temps }\end{array}$ & $\begin{array}{c}\text { Taux } \\
\text { d'amor- } \\
\text { tissement }\end{array}$ & $\begin{array}{c}\text { Nombre de } \\
\text { modes }\end{array}$ & $\begin{array}{c}\text { Bornes } \\
\text { d'intégration de } \\
\text { l'ELB-CA }\end{array}$ \\
\hline$x_{0}(\mathrm{~m})$ & $t_{0}(\mathrm{~s})$ & $t_{\mathrm{f}}(\mathrm{s})$ & $N_{\mathrm{t}}$ & $\xi_{k}=\xi(\%)$ & $N$ & $a(\mathrm{~m})$ & $b(\mathrm{~m})$ \\
\hline 0,3 & 0 & 15 & 7500 & 0,5 & 10 & 0 & 0,5 \\
\hline
\end{tabular}

Tableau 3. Fréquences des ELB-CA en $h / 2$ et sur l'axe neutre pour le cas L, resp. sur l'intervalle [0-100] Hz et [0-50] Hz.

\begin{tabular}{cccccc}
\hline Fréquences & ELB $h / 2(\mathrm{~Hz})$ & Fréquences & ELB axe $(\mathrm{Hz})$ & Combinaison & Fréquences ELB $h / 2(\mathrm{~Hz})$ \\
\hline$f_{1}$ & 7,11 & $f_{1}^{\text {axe }}$ & 12,51 & $f_{2}-f_{1}$ & 12,49 \\
$f_{\text {sup } 1}$ & 12,51 & $f_{2}^{\text {axe }}$ & 14,21 & $2 \times f_{1}$ & 14,22 \\
$f_{\text {sup } 2}$ & 14,21 & $f_{3}^{\text {axe }}$ & 18,86 & $f_{3}-f_{2}$ & 18,83 \\
$f_{2}$ & 19,60 & $f_{4}^{\text {axe }}$ & 26,70 & $f_{2}+f_{1}$ & 26,71 \\
$f_{\text {sup } 3}$ & 26,70 & $f_{5}^{\text {axe }}$ & 31,45 & $f_{5}-f_{4}$ & 31,33 \\
$f_{3}$ & 38,43 & $f_{6}^{\text {axe }}$ & 39,19 & $2 \times f_{2}$ & 39,20 \\
$f_{4}$ & 63,53 & $f_{7}^{\text {axe }}$ & 44,03 & $f_{4}-f_{2}$ & 43,93 \\
$f_{5}$ & 94,86 & - & - & - & - \\
\hline
\end{tabular}

TF ELB-CA axe

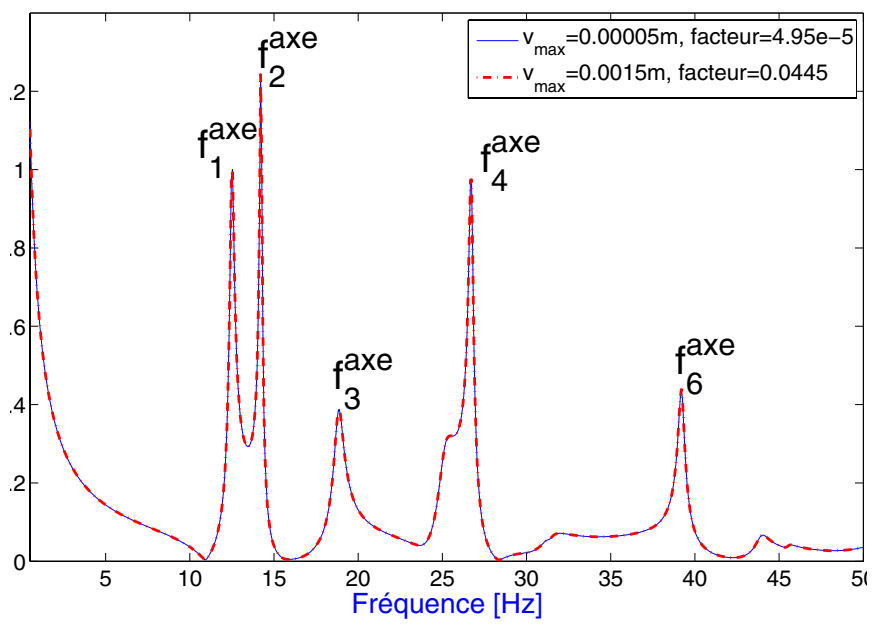

Fig. 4. TF normalisée d'un ELB-CA situé au niveau de l'axe neutre dans le cas $\mathrm{L}$ pour une flèche maximale de la poutre $v_{\max }=5 \times 10^{-5} \mathrm{~m}$ et $v_{\max }=1,5 \times 10^{-3} \mathrm{~m}$.

$\left.f_{2}-f_{1}, f_{2}+f_{1}, \ldots\right)$ et l'amplitude de leur pic dépend de l'importance des amplitudes de déformation atteintes.

L'étude de la mesure de l'ELB-CA situé au niveau de l'axe neutre va permettre de donner une explication quant à l'apparition de ces fréquences additionnelles.

\subsubsection{ELB-CA situé au niveau de l'axe neutre $(d=0)$}

Les mesures simulées de l'ELB-CA situé au niveau de l'axe neutre sont calculées à partir de l'équation (24); leurs TF sont ensuite déterminées. L'ELB-CA est placé entre les mêmes abscisses de la poutre et les mesures sont effectuées entre les instants $t_{0}=0$ et $t_{\mathrm{f}}$, intervalle de temps discrétisé en $N_{\mathrm{t}}$ points. Les deux valeurs de la flèche maximale de la poutre E-E envisagées sont les mêmes que précédemment à savoir $v_{\max }=5 \times 10^{-5} \mathrm{~m}$ (flèche faible) et $v_{\max }=1,5 \times 10^{-3} \mathrm{~m}$ (flèche plus importante).

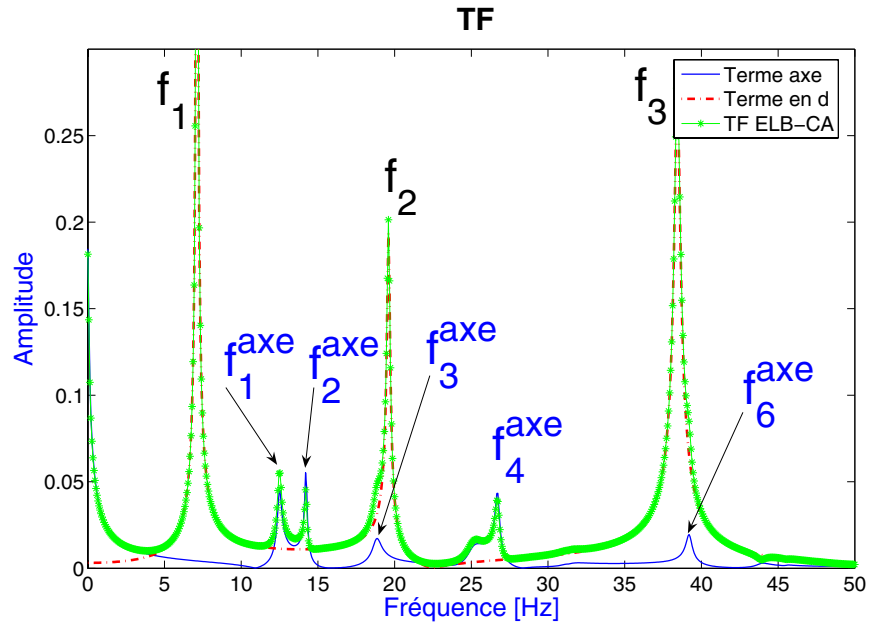

Fig. 5. Comparaison des TF des termes composant la mesure de l'ELB-CA dans le cas L pour une flèche maximale de la poutre $v_{\max }=1,5 \times 10^{-3} \mathrm{~m}$.

Sur la figure 4, les TF de l'ELB-CA situé au niveau de l'axe neutre ont été tracées pour les deux niveaux de flèche, en les normalisant au premier pic de fréquence (le facteur de normalisation est de $4,95 \times 10^{-5}$ pour $v_{\max }=$ $5 \times 10^{-5} \mathrm{~m}$ et de 0,045 pour $v_{\max }=1,5 \times 10^{-3} \mathrm{~m}$ ). Les TF se superposent parfaitement et les fréquences observées sur ces spectres, répertoriées dans le tableau 3, ne sont pas les fréquences de vibration de flexion de la poutre mais des combinaisons linéaires de celles-ci.

Les rapports d'amplitude (par rapport au premier niveau de flèche, $\left.v_{\max }=5 \times 10^{-5} \mathrm{~m}\right)$ du premier pic de la TF de l'ELB-CA en $h / 2\left(f_{1}\right)$ et du deuxième pic de la TF de l'ELB-CA au niveau de l'axe neutre $\left(f_{2}^{\text {axe }}\right)$ sont répertoriés dans le tableau 5 , en fonction de trois niveaux de la flèche maximale de la poutre (les deux déjà cités plus un niveau intermédiaire pour $\left.v_{\max }=5 \times 10^{-4} \mathrm{~m}\right)$. Les résultats du tableau montrent que l'amplitude des pics de fréquences pour l'ELB-CA au niveau de l'axe neutre 
n'évolue pas de façon linéaire, comme c'est le cas pour l'ELB-CA en $h / 2$, mais beaucoup plus rapidement.

Sur la figure 5 sont tracées les TF des termes composant la mesure de l'ELB-CA en $h / 2$ (26), soit à la surface de la poutre. Il apparaît sur cette figure que les fréquences observées sur la TF du terme de la mesure de l'ELB-CA en $h / 2$ dépendant de la distance à l'axe neutre $d$ (« terme en $d »)$, sont les fréquences de vibration de flexion de la poutre. L'apparition des pics supplémentaires au niveau de la TF de l'ELB-CA en $h / 2$ peut alors s'expliquer par l'augmentation rapide de l'amplitude des pics de la TF de l'ELB-CA situé au niveau de l'axe neutre (Tab. 5), qui tend à se rapprocher, lorsque la flèche de la poutre augmente, de l'ordre de grandeur de l'amplitude des pics de la TF du «terme en $d »$. Ainsi, les fréquences additionnelles qui apparaissent au niveau de la mesure de l'ELB-CA en $h / 2$ (Fig. 3b) proviennent uniquement de la mesure de l'ELB-CA situé au niveau de l'axe neutre et ce sont celles qui ont l'amplitude la plus importante sur la TF de ce dernier (Fig. 4), c'est-à-dire $f_{1}^{\text {axe }}, f_{2}^{\text {axe }}, f_{3}^{\text {axe }}, f_{4}^{\text {axe }}$ et $f_{6}^{\text {axe }}$. Les pics des fréquences $f_{3}^{\text {axe }}$ et $f_{6}^{\text {axe }}$ ne sont pas visibles au niveau de la TF de l'ELB-CA situé en $h / 2$ car leurs amplitudes sont plus faibles et les fréquences propres de la structure $\left(f_{2}\right.$ et $\left.f_{3}\right)$ qui les masquent complètement.

Nous allons maintenant étudier la mesure de l'ELBCA obtenue à partir du système d'équations couplées et non-linéaires (17), car pour des niveaux de flèche importants les équations du cas L ne sont plus valables. Ainsi la même étude va être effectuée pour le cas $\mathrm{G}$ afin de voir si l'on retrouve des résultats similaires à ceux obtenus précédemment.

\subsection{Cas général}

L'hypothèse $\mathrm{H} 2$ est effectuée pour le calcul numérique de la mesure de l'ELB-CA dans cette partie. Les équations aux dérivées partielles du système (17) sont discrétisées en espace par la méthode des éléments-finis et les équations différentielles en temps obtenues sont résolues par la méthode de Runge Kutta. La procédure est détaillée dans [29]. La poutre est discrétisée en $N_{\mathrm{e}}=30$ éléments. Le choc est modélisé par une demi-sinusoïde dont la durée est $t_{\text {choc }}=0,004 \mathrm{~s}$. Les paramètres de l'étude numérique sont les mêmes qu'en section 3.1, notamment le placement de l'ELB-CA, les instants $t_{0}=0$ et $t_{\mathrm{f}}$ des mesures (intervalle de temps discrétisé en $N_{\mathrm{t}}$ points) et le nombre de modes $N=10$ (les 5 premiers modes transverses et axiaux). L'ensemble des paramètres est répertorié dans le tableau 2. Les déplacements axial et transverse de la poutre, resp. $u$ et $v$, ainsi obtenus sont réinjectés dans l'équation (18) pour déterminer la mesure de l'ELB-CA à la surface de la poutre $(d=h / 2)$ et dans l'équation (19) pour celle de l'ELB-CA au niveau de l'axe neutre.

Comme pour le cas Linéaire (cas L), les deux mêmes valeurs de la flèche maximale $v_{\max }$ de la poutre E-E ont été choisies. La figure 6 a est obtenue pour $v_{\max }=5 \times$ $10^{-5} \mathrm{~m}$ (faible flèche) et la figure $6 \mathrm{~b}$ correspond à $v_{\max }=$ $1,5 \times 10^{-3} \mathrm{~m}$ (flèche plus importante, $v_{\max } / h=0,5$ ). (a)

TF ELB - en $\mathrm{h} / 2$

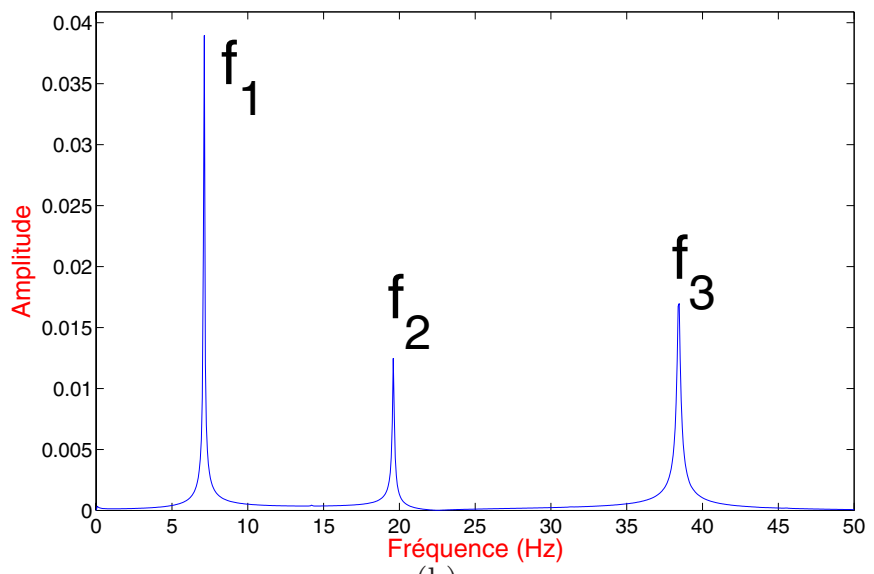

(b)

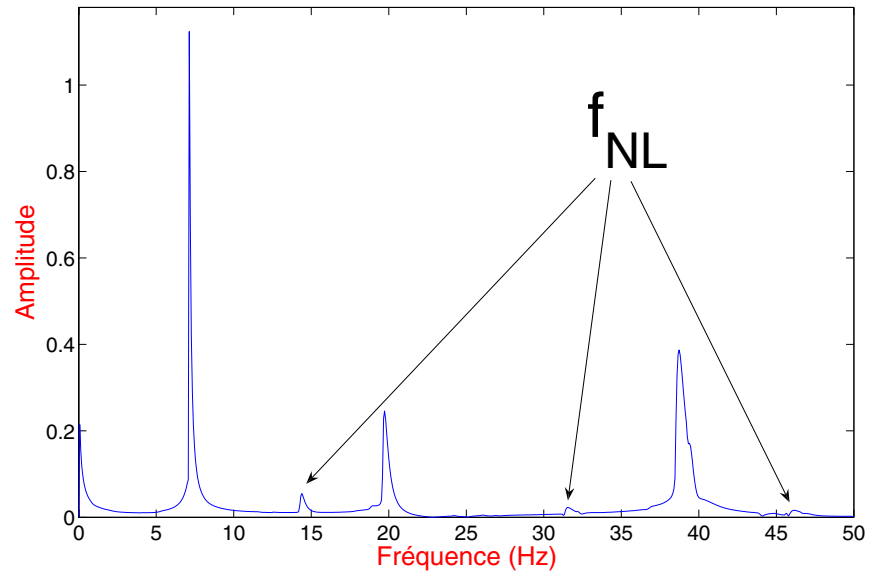

Fig. 6. TF d'un ELB-CA situé à la surface de la poutre $(d=$ $h / 2$ ) dans le cas $\mathrm{G}$ pour une flèche maximale de la poutre (a) $v_{\max }=5 \times 10^{-5} \mathrm{~m}$ et (b) $v_{\max }=1,5 \times 10^{-3} \mathrm{~m}$.

Les graphes de la figure 6 paraissent similaires, à première vue, à ceux du cas L (Fig. 3). En effet, des pics supplémentaires apparaissent lorsque la flèche augmente et l'amplitude de ces pics est du même ordre de grandeur que pour le cas L (Fig. 3). Cependant les fréquences des pics supplémentaires, répertoriées dans le tableau 4, sont différentes.

L'allure des pics de la TF, lorsque la flèche de la poutre augmente, diffère également du cas L. En effet, ils sont dissymétriques (la pente est plus raide à gauche des pics qu'à droite (Fig. 6b)), ce qui est significatif du caractère non linéaire d'une structure et que vient confirmer une analyse de ces pics par la transformée en ondelettes continue présentée dans [30]. Sur la figure 7, les transformées en ondelettes du premier mode de la mesure de l'ELB-CA en $h / 2$ sont présentées pour les deux niveaux de flèche. Les graphes présentent l'évolution de la fréquence en fonction du temps ou fréquence instantanée, la courbe représentant l'arête de la transformée en ondelettes.

Pour la première valeur de flèche, $v_{\max }=5 \times 10^{-5} \mathrm{~m}$, la fréquence est constante en fonction du temps et donc 
Tableau 4. Fréquences des ELB-CA en $h / 2$ et sur l'axe neutre pour le cas G, resp. sur l'intervalle [0-100] Hz et [0-50] Hz.

\begin{tabular}{cccccc}
\hline \multicolumn{2}{c}{ Fréquences ELB $\mathrm{h} / 2(\mathrm{~Hz})$} & \multicolumn{2}{c}{ Fréquences ELB axe $(\mathrm{Hz})$} & Combinaison Fréquences ELB h/2 $(\mathrm{Hz})$ \\
\hline$f_{1}$ & 7,11 & $f_{1}^{\text {axe }}$ & 12,46 & $f_{2}-f_{1}$ & 12,49 \\
$f_{N L 1}$ & 14,39 & $f_{2}^{\text {axe }}$ & 14,22 & $2 \times f_{1}$ & 14,22 \\
$f_{2}$ & 19,60 & $f_{3}^{\text {axe }}$ & 18,80 & $f_{3}-f_{2}$ & 18,83 \\
$f_{N L 2}$ & 31,55 & $f_{4}^{\text {axe }}$ & 25,24 & $f_{4}-f_{3}$ & 25,10 \\
$f_{3}$ & 38,43 & $f_{5}^{\text {axe }}$ & 26,76 & $f_{2}+f_{1}$ & 26,71 \\
$f_{N L 3}$ & 46,1 & $f_{6}^{\text {axe }}$ & 31,31 & $f_{5}-f_{4}$ & 31,17 \\
$f_{4}$ & 63,53 & $f_{7}^{\text {axe }}$ & 39,21 & $2 \times f_{2}$ & 39,20 \\
$f_{5}$ & 94,87 & $f_{8}^{\text {axe }}$ & 43,97 & $f_{4}-f_{2}$ & 43,93 \\
- & - & $f_{9}^{\text {axe }}$ & 45,53 & $f_{3}+f_{1}$ & 45,54 \\
\hline
\end{tabular}

(a)

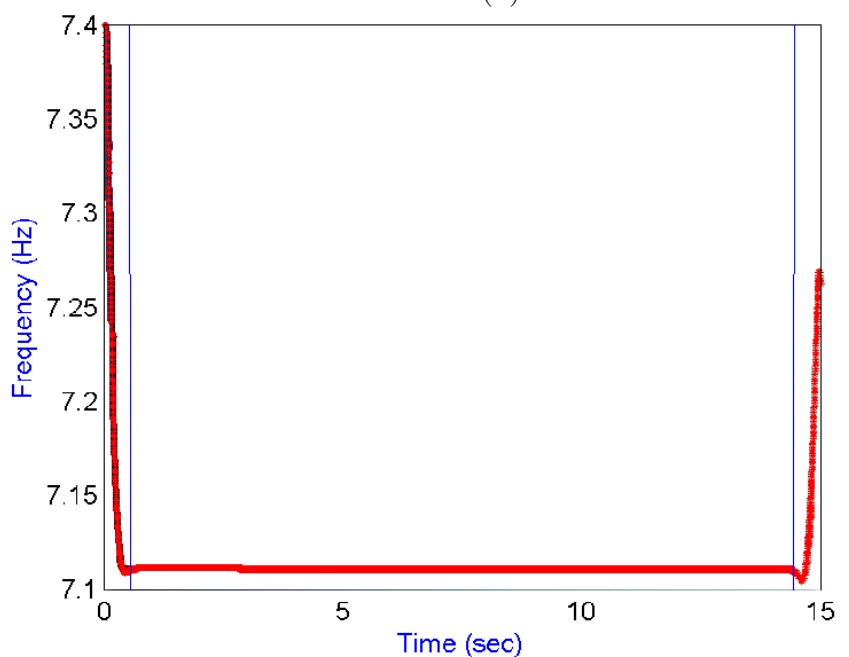

(b)

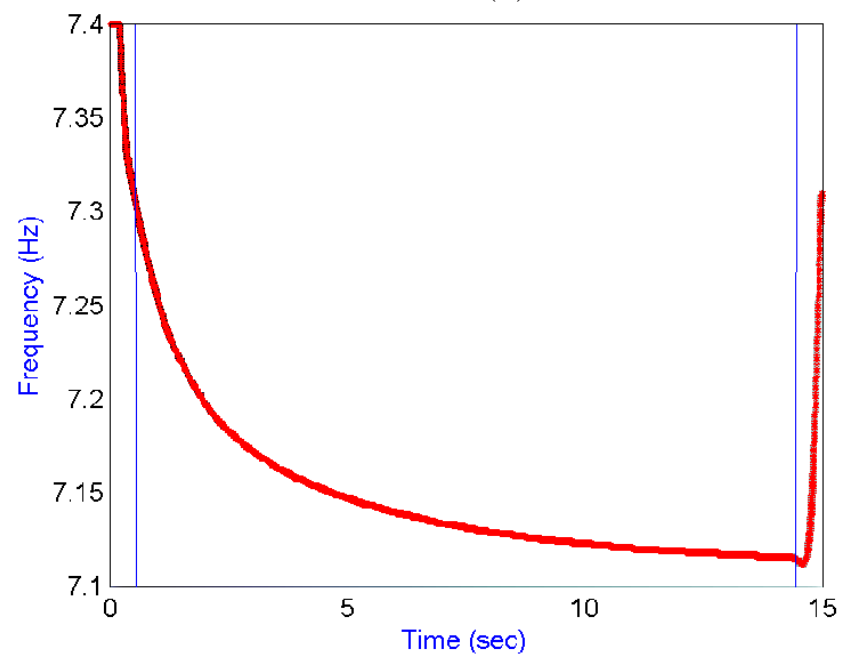

Fig. 7. Transformée en ondelettes du premier mode de la TF de l'ELB-CA situé à la surface de la poutre $(d=h / 2)$ dans le cas $\mathrm{G}$ pour une flèche maximale de la poutre (a) $v_{\max }=$ $5 \times 10^{-5} \mathrm{~m}$ et (b) $v_{\max }=1,5 \times 10^{-3} \mathrm{~m}$.

l'évolution du système est quasi-linéaire. Les fortes pentes aux extrémités du graphique proviennent de l'effet de bord et ne sont pas à prendre en compte. Pour le niveau de flèche plus important, $v_{\max }=1,5 \times 10^{-3} \mathrm{~m}$, la fréquence varie sensiblement avec le temps, ce qui indique
TF ELB-CA axe

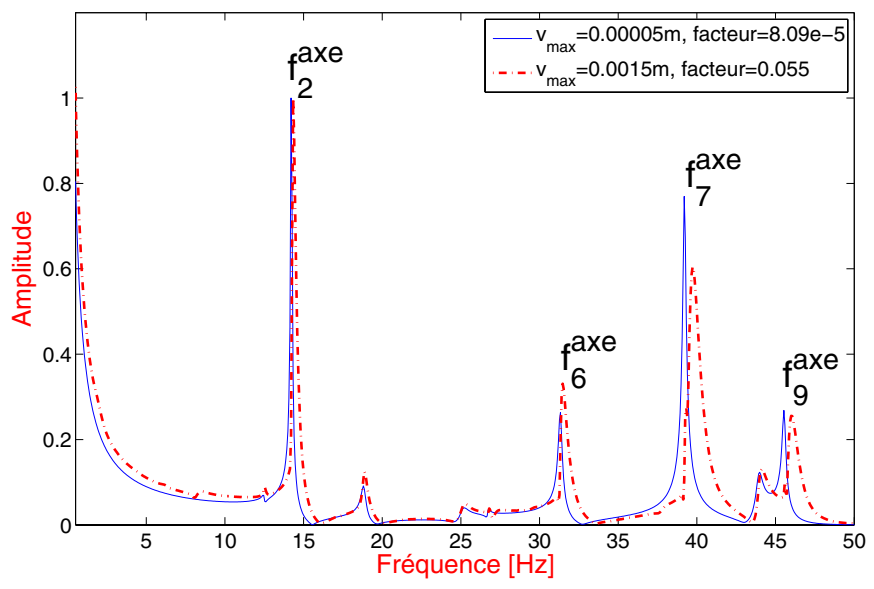

Fig. 8. TF normalisée d'un ELB-CA situé au niveau de l'axe neutre dans le cas $G$, pour une flèche maximale de la poutre $v_{\max }=5 \times 10^{-5} \mathrm{~m}$ et $v_{\max }=1,5 \times 10^{-3} \mathrm{~m}$.

un comportement non-linéaire de la poutre. Pour le cas $\mathrm{L}$, les pics des TF ont toujours la même allure symétrique et une transformée en ondelettes des modes issus de la mesure des ELB-CA donnerait un résultat similaire à celui de la figure 7a (fréquence instantanée contante, évolution linéaire) et ce quel que soit le niveau de flèche de la poutre.

Dans le tableau 5, les rapports d'amplitudes (suivant le premier niveau de flèche, $v_{\max }=5 \times 10^{-5} \mathrm{~m}$ ) du premier pic pour l'ELB-CA en $h / 2\left(f_{1}\right)$ et du deuxième pic pour l'ELB-CA au niveau de l'axe neutre $\left(f_{2}^{\text {axe }}\right)$ ont été répertoriés en fonction de la flèche. Si la variation d'amplitude est à peu près linéaire pour l'ELB-CA en $h / 2$, elle ne l'est plus pour l'ELB-CA situé au niveau de l'axe neutre où elle augmente rapidement avec la flèche maximale de la poutre. L'apparition des pics supplémentaires au niveau de l'ELB-CA à la surface de la poutre s'explique alors de la même manière que pour le cas L, à l'aide de la figure 9 où sont tracées les TF des termes composant la mesure de l'ELB-CA situé à la surface de la poutre (26). Les fréquences additionnelles qui ressortent au niveau de la mesure de l'ELB-CA en $h / 2$ (Fig. 6b) sont celles qui ont l'amplitude la plus importante sur le spectre de l'ELB-CA situé au niveau de l'axe neutre (Fig. 8 et Tab. 4$), f_{2}^{\text {axe }}, f_{6}^{\text {axe }}, f_{7}^{\text {axe }}$ et $f_{9}^{\text {axe }}$ (le pic supplémentaire dû à $f_{7}^{\text {axe }}$ n'apparaît pas sur la figure $6 \mathrm{~b}$ car il est masqué par 
Tableau 5. Variations de l'amplitude des pics de la première fréquence pour l'ELB-CA en $h / 2\left(f_{1}\right)$ et de la deuxième fréquence pour l'ELB-CA sur l'axe neutre $\left(f_{2}^{\text {axe }}\right)$ en fonction de la flèche maximale de la poutre pour les cas L et G.

\begin{tabular}{ccccc}
\hline $\begin{array}{c}\text { Flèche } \\
\text { maximale }(\mathrm{m})\end{array}$ & \multicolumn{2}{c}{ Amplitude - cas L } & \multicolumn{2}{c}{ Amplitude - cas G } \\
\cline { 2 - 5 } & pic $f_{1}$ & pic $f_{2}^{\text {axe }}$ & pic $f_{1}$ & pic $f_{2}^{\text {axe }}$ \\
\hline $5 \times 10^{-5}$ & 0,0175 & $4,61 \times 10^{-5}$ & 0,039 & $8,09 \times 10^{-5}$ \\
$5 \times 10^{-4} / 5 \times 10^{-5}$ & 10 & 107 & 10,5 & 89 \\
$1,5 \times 10^{-3} / 5 \times 10^{-5}$ & 30 & 976 & 28,8 & 680 \\
\hline
\end{tabular}

\section{TF}

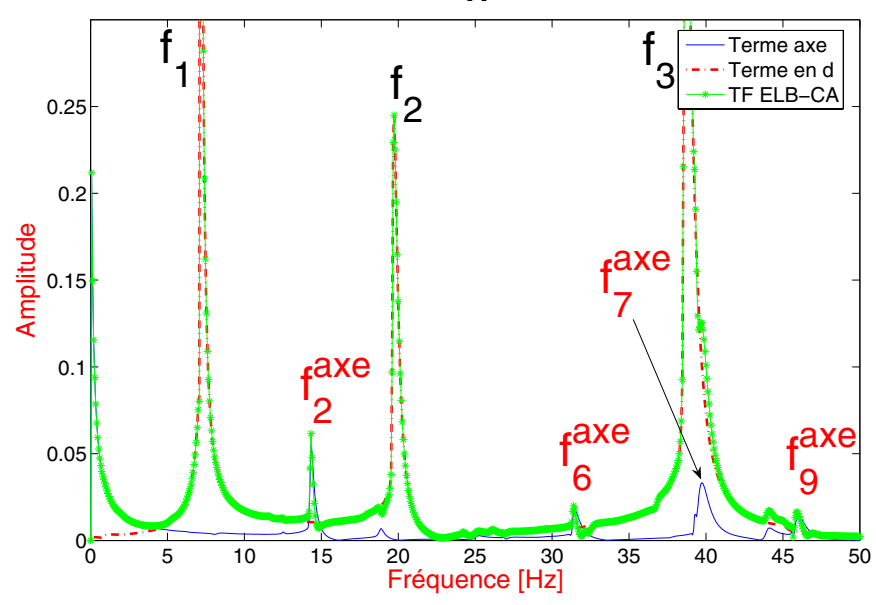

Fig. 9. Comparaison des TF des termes composant la mesure de l'ELB-CA dans le cas $G$ pour une flèche maximale de la poutre $v_{\max }=1,5 \times 10^{-3} \mathrm{~m}$.

$f_{3}$, troisième fréquence propre de la structure, c'est-à-dire très proche en valeur).

Les résultats obtenus lors de l'étude du cas $\mathrm{G}$ sont similaires à ceux issus de l'étude du cas L concernant l'apparition des pics supplémentaires. Cependant, les fréquences de ces pics ne sont pas les mêmes et l'allure du spectre de l'ELB-CA situé au niveau de l'axe neutre est différente pour les deux modèles (Figs. 4 et 8), et ce même pour un faible niveau de flèche. La seule différence entre les mesures de l'ELB-CA au niveau de l'axe neutre pour les deux cas L (Éq. (24)) et G (Éq. (19)) est le terme dépendant de $u^{\prime}$, ce qui tend à montrer que, dans le cas présent, le couplage entre les déplacements axial et transverse est toujours présent, même pour de faibles valeurs de flèche. De ce fait, le découplage des équations menant au système (17) ne peut se faire et l'hypothèse du cas L est trop restrictive. Il est donc nécessaire d'utiliser le cas G pour déterminer la mesure réalisée par un ELB-CA.

\section{Conclusion}

Les formules usuelles de la littérature utilisent la mesure de l'ELB en tant que telle, sans regarder le détail des paramètres physiques qui la compose, notamment les champs de déplacement (axial et transverse dans notre étude). Dans cet article, les formulations analytiques de la réponse dynamique des Extensomètres Longue Base
Continûment Attachés (ELB-CA) sont présentées dans le cas d'une poutre d'Euler-Bernoulli en vibration de flexion. Deux cas ont été proposés pour déterminer la mesure réalisée par un ELB-CA : l'un avec les équations du mouvement de la poutre découplées et linéaires, et l'autre avec les équations couplées et non-linéaires. Des simulations numériques ont été réalisées dans le cas de la réponse vibratoire d'une poutre bi-encastrée à un choc pour différentes valeurs de flèche et avec un ELB-CA situé à la surface et au niveau de l'axe neutre de celle-ci.

Lorsque la flèche maximale de la poutre augmente, favorisant ainsi l'apparition de non-linéarités géométriques, la transformée de Fourier des mesures de l'ELB-CA contient les fréquences propres de la structure, auxquelles viennent s'ajouter des fréquences additionnelles. Ces fréquences supplémentaires sont des combinaisons de celles de la structure.

Des résultats similaires ont été obtenus dans les deux cas d'étude : équations découplées-linéaires et équations couplées-non-linéaires. L'apparition de pics supplémentaires sur la $\mathrm{TF}$ de la mesure réalisée par l'ELB-CA est expliquée en analysant la TF issue de la mesure de l'ELB-CA situé au niveau de l'axe neutre. De plus, la comparaison des deux cas de l'étude montre que l'utilisation du système d'équations découplées pour décrire le mouvement de la poutre est trop restrictive, du fait du couplage entre les déplacements axial et transverse même pour de faibles niveaux de la flèche maximale de la poutre.

D'autres simulations numériques tendent à montrer que les conditions aux limites, la longueur du capteur ainsi que son placement sont des paramètres importants quant à l'apparition des pics supplémentaires, dûs aux non-linéarités géométriques, au niveau des TF de la mesure réalisée par l'ELB-CA.

Par ailleurs, une validation expérimentale de ces résultats numériques est en cours de réalisation.

\section{Références}

[1] J.M. Ko, Y.Q. Ni, Technology developments in structural health monitoring of large-scale bridges, Eng. Structures 27 (2005) 1715-1725

[2] V. Lekidis, M. Tsakiri, K. Makra, C. Karakostas, N. Klimis, I. Sous, Evaluation of dynamic response and local soil effects of the Evripos cable-stayed bridge using multisensor monitoring systems, Eng. Geology 79 (2005) 43-59

[3] W.X. Ren, X.L. Peng, Y.Q. Lin, Experimental and analytical studies on dynamic characteristics of a large span cable-stayed bridge, Eng. Structures 27 (2005) 535-548 
[4] M. Meo, G. Zumpano, On the optimal sensor placement techniques for a bridge structure, Eng. Structures 27 (2005) 1488-1497

[5] T.H. Yan, R.M. Lin, General optimization of sizes or placement for various sensors/actuators in structure testing and control, Smart Materials and Structures 15 (2006) $724-736$

[6] D. Inaudi, State of the art in fiber optic sensing technology and EU structural health monitoring projects, in Proc. of the First International Conference on Structural Health Monitoring and Intelligent Infrastructure, Tokyo, Japan, 2003

[7] Y. Zhao, F. Ansari, Quasi-distributed fiber-optic strain sensor: principle and experiment, Appl. Opt. 40 (2001) 3176-3181

[8] SMARTEC (http://www.smartec.ch), SOFO Products (Standard Deformation Sensor, SMARTape Deformation Sensor and Dynamic Reading Unit)

[9] FOGALE (http://www.fogale.fr), Product: LISE SYSTEM, OCT-EXTEND.

[10] FOX-TEK (http://www.fox-tek.com)

[11] D. Inaudi, S. Vurpillot, Monitoring of Concrete Bridges with Long-Gage Fiber Optic Sensors, J. Intell. Mtals. Sys. Struct. 10 (1999) 280-292

[12] D. Inaudi, B. Glisic, Combining Static and Dynamic deformation monitoring with long-gauge fiber optic sensors, in Proceedings of the International Association for Bridge Maintenance and Safety, Kyoto, Japan, 2004

[13] E. Reynders, G. De Roeck, Measurement of modal curvature using optical fiber strain sensors and application to damage identification using vibration monitoring, in Proceedings of the 17th International Conference on Optical Fiber Sensors, 2005, pp 1076-1079

[14] B. Glisic, D. Inaudi, Sensing tape for easy integration of optical fiber sensors in composite structures, in Proceedings of the 16th International Conference on Optical Fiber Sensors, Nara, Japan, 2003

[15] A. Courteville, M. Delaveau, S. Delepine-Lesoille, E. Merliot, L. Quetel, New concept of low intrusion quasidistributed optical fibre extensometer, in Proceedings of Photonics Europe, 2006

[16] F. Casciati, M. Domaneschi, D. Inaudi, A. Figini, B. Glisic, A. Gupta, Long-gauge fiber optic sensors: a new approach to dynamic system identification, in Proceedings of the Third European Conference on Structural Control, 2004

[17] F. Bourquin, Towards Modal Fiber Optical Sensors for Slender Beams, in Proceedings of the 2nd European
Workshop on Structural Health Monitoring, Munich, Germany, 2004, pp. 1193-1198

[18] J.M. Lopez-Higuera, Handbook of Optical Fibre Sensing Technology, John Wiley \& Sons, New York, 2002

[19] F. Ansari, Y. Libo, Mechanics of Bond and Interface Shear Transfer in Optical Fiber Sensors, J. Eng. Mech. 124 (1998) 385-394

[20] J.P. Perez, Optique : fondements et applications avec 250 exercices et problèmes résolus, Dunod, Paris, 2004

[21] J.S. Sirkis, H.W. Haslach, Interferometric Strain Measurement by Arbitrarily Configured, SurfaceMounted, Optical Fibers, J. Lightwave Technology 10 (1990) 1497-1503

[22] T.G. Giallorenzi, J.A. Bucaro, A. Dandrige, G.H. Sigel, J.H. Cole, S.C. Rashleigh, R.G. Priest, Optical Fiber Sensor Technology, J. Quantum Electronics 4 (1982) 626665

[23] R. Deltheil, Cours de mathématiques générales : IIÉléments de calcul intégral : Applications géométriques de l'analyse, Baillière, 1947

[24] M. Géradin, D. Rixen, Théorie des vibrations : Application à la dynamique des structures, Masson, 1996

[25] H. Park, H.S. Jung, Y.H. Kwon, J.H. Seo, Mathematical models for assessment of the safety of steel beams based on average strains from long gage optic sensors, Sensors and Actuators A: Physical 125 (2006) 109-113

[26] S. Delepine-Lesoille, E. Merliot, C. Boulay, L. Quétel, M. Delaveau, A. Courteville, Quasi-distributed optical fibre extensometers for continuous embedding into concrete: design and realization, Smart Materials and Structures 15 (2006) 931-938

[27] Y. Zhao, F. Ansari, Quasi-distributed white light fiber optic strain sensor, Optics communication 196 (2001) 133137

[28] S. Delepine-Lesoille, M. Quiertant, E. Merliot, Y. Gautier, J. Dumoulin, S. Dubroca, L. Quétel, M. Delaveau, A. Courteville, Quasi-distributed optical fibre extensometers for continuous embedding into concrete: Design, Realization, Laboratory and Field Tests, in Proceedings of the Third European Workshop on Structural Health monitoring, Grenade, Spain, 2006, pp. 946-953

[29] T.M. Nguyen, Dynamique non linéaire des systèmes mécaniques couplés : Réduction de modèle et Identification, Thèse, ENPC-MODES, 2006

[30] T.P. Le, P. Argoul, Continuous wavelet transform for modal identification using free decay response, J. Sound Vib. 277 (2004) 73-100 